\title{
Antiferromagnetic coupling supported by metallophilic interactions. Theoretical view
}

\author{
Zoraida Sandoval-Olivares ${ }^{1}$, Eduardo Solis-Céspedes ${ }^{2,3}$, Dayán Páez-Hernández ${ }^{* 4,5}$
}

December 22, 2021

\footnotetext{
${ }^{1}$ Doctorado en Fisicoquímica Molecular, Universidad Andrés Bello, República 275, Santiago, Chile. z.sandoval.o@gmail.com ${ }^{2}$ Escuela de Bioingeniería Médica, Facultad de Medicina, Universidad Católica del Maule, Chile. esolis@ucm.cl

${ }^{3}$ Laboratorio de Bioinformática y Química Computacional, Facultad de Medicina, Universidad Católica del Maule, Chile.

${ }^{4}$ Center of Applied Nanoscience (CANS), Universidad Andres Bello, República 330, Santiago, Chile. dayan.paez@unab.cl

${ }^{5}$ Departamento de Ciencias Químicas, Universidad Andres Bello, República 275, Santiago, Chile.
}

\section{Electronic Supporting Information}

\section{Monomers information}

1. Molecular structure of the monomeric units obtained from the geometry optimization of the dimers.

2. Occupation numbers of the active orbitals obtained from $\operatorname{CAS}(7,5)$ and $\operatorname{CAS}(11,7)$.

3. Optimized geometry parameters

\section{Dimers information}

1. Geometrical parameters obtained from the optimized structure of the dimers.

2. Morokuma-Ziegler energy decomposition scan at different Pt...Pt distances.

3. Evolution of the molecular orbitals in the A irreducible representation at different Pt...Pt distances.

4. Calculated J parameter at Broken-Symmetry level of theory considering different contributions of the Hartree-Fock exchange.

5. Cartesian coordinates of the optimized complexes. 


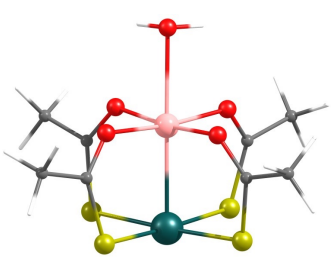

a) $\mathrm{PtCo}(\mathrm{SAc})_{4}\left(\mathrm{H}_{2} \mathrm{O}\right)$

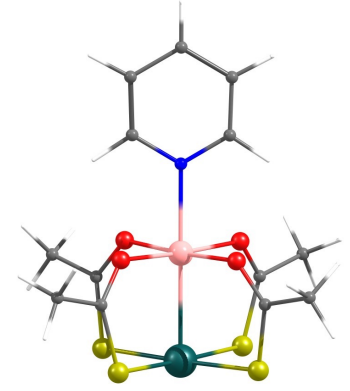

b) $\mathrm{PtCo}(\mathrm{SAc})_{4}(\mathrm{py})$

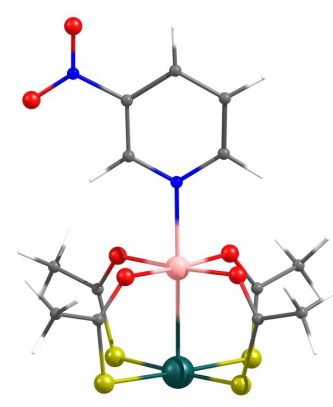

c) $\mathrm{PtCo}(\mathrm{SAc})_{4}\left(3-\mathrm{NO}_{2}\right.$ py $)$

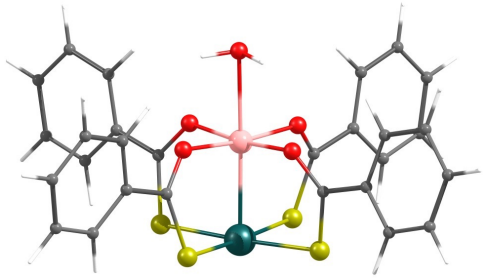

d) $\mathrm{PtCo}(\mathrm{tba})_{4}\left(\mathrm{H}_{2} \mathrm{O}\right)$

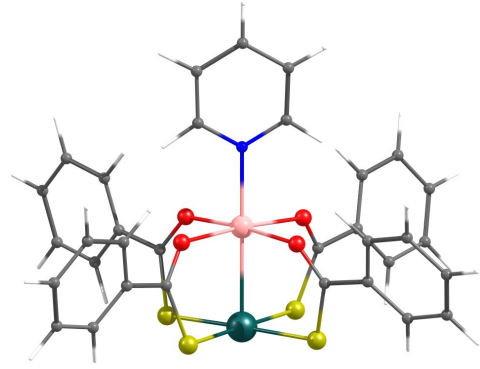

e) $\mathrm{PtCo}(\mathrm{tba})_{4}(\mathrm{py})$

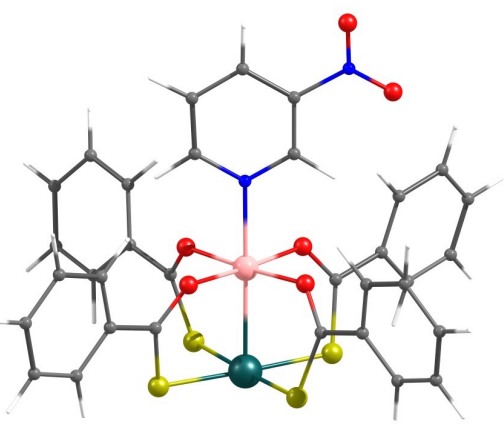

f) $\mathrm{PtCo}(\mathrm{tba})_{4}\left(3-\mathrm{NO}_{2}\right.$ py $)$

Figure S1: Molecular structure for the monomeric units obtained from the optimized geometries of the dimers $\left[\mathrm{PtCo}(X)_{4}(Y)\right]_{2}$, where $\mathrm{X}=\mathrm{SAc}$, tba and $\mathrm{Y}=\mathrm{H}_{2} \mathrm{O}, 3-\mathrm{NO}_{2}$ py, py. 

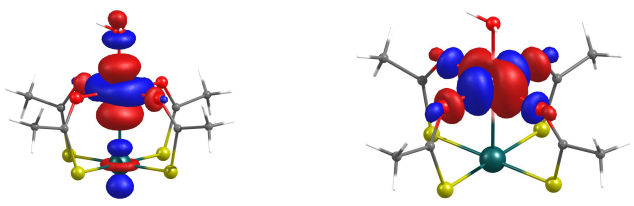
a) $1.340 \quad 1.455$
b) 1.504
1.483
$1.180 \quad 1.356$
c) 1.310
1.434
1.455
1.485
d) 1.296
1.355
$1.193 \quad 1.344$
e) 1.312
1.326
$1.285 \quad 1.294$
f) 1.278
1.355
1.222
1.282
$1.281 \quad 1.291$
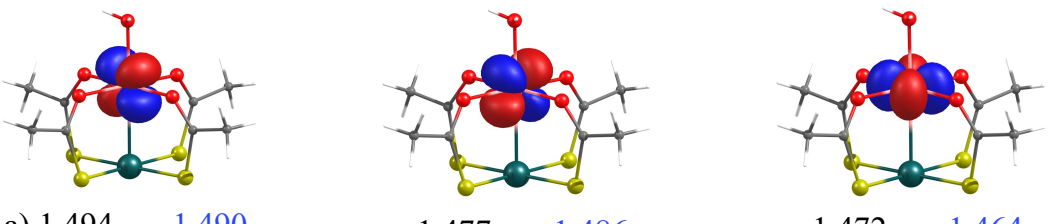
a) 1.494
1.490
b) 1.294
1.489
d) $1.495 \quad 1.529$
e) $1.486 \quad 1.540$
f) $1.496 \quad 1.531$

1.477

1.486

$1.292 \quad 1.489$

$1.487 \quad 1.470$

$1.491 \quad 1.514$

1.524

1.454

1.464

$1.492 \quad 1.517$

$1.469 \quad 1.459$

$1.470 \quad 1.474$

$1.494 \quad 1.495$

$1.476 \quad 1.473$
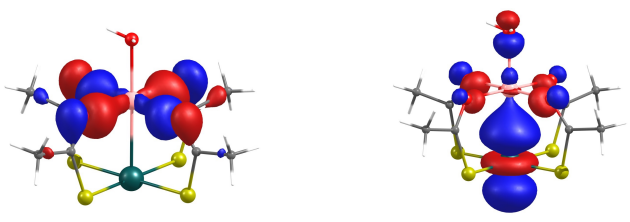
a) 1.924
1.825
b) 1.784
1.781
c) 1.975
1.774
d) 1.999
1.833
e) 1.967
1.867
f) 1.998
1.833

Figure S2: Occupations numbers of the active orbitals obtained for monomeric units at CAS(7,5) (black) and CAS(11,7) (blue) level. For a correct interpretation it is necessary to associate the label in the first column with Figure S1. 
Table S1: Optimized geometrical parameters $(\AA)$ obtained for monomers.

\begin{tabular}{cccc}
\hline Molecule & $\mathrm{d}\left(\mathrm{L}_{a x}-\mathrm{Co}\right)$ & $\mathrm{d}(\mathrm{Co}-\mathrm{Pt})$ & $\mathrm{d}\left(\mathrm{Co}-\mathrm{O}_{e q}\right)$ \\
\hline PtCo $(\mathrm{SAc})_{4}\left(\mathrm{OH}_{2}\right)$ & 2.082 & 2.634 & 2.052 \\
& & & \\
PtCo $(\mathrm{SAc})_{4}\left(3-\mathrm{NO}_{2} p y\right)$ & 2.114 & 2.635 & 2.052 \\
& & & \\
$\mathrm{PtCo}(t b a)_{4}\left(\mathrm{OH}_{2}\right)$ & 2.051 & 2.569 & 2.108 \\
& $(2.035)$ & $(2.552)$ & $(2.069)^{a}$ \\
$\mathrm{PtCo}(t b a)_{4}\left(3-\mathrm{NO}_{2} p y\right)$ & 2.114 & 2.569 & 2.108 \\
& & & \\
{$\left[\mathrm{PtCo}(\mathrm{SAc})_{4}(\mathrm{py})\right]$} & 2.140 & 2.682 & 2.100 \\
& $(2.101)$ & $(2.630)$ & $(2.081)^{b}$ \\
$\mathrm{PtCo}(t b a)_{4}(p y)$ & 2.139 & 2.647 & 2.099 \\
& & & \\
\hline
\end{tabular}

Experimental data in parenthesis obtained from ${ }^{a}$ Chem. Sci. 2012, 3, 602-609 ${ }^{b}$ Inorg. Chem. 2013, 52, 4926-4933.

Table S2: Optimized geometrical parameters $(\AA)$ obtained for dimers.

\begin{tabular}{cccccc}
\hline Molecule & $\mathrm{d}(\mathrm{Pt}-\mathrm{Pt})$ & $\mathrm{d}(\mathrm{M}-\mathrm{Pt})$ & $\mathrm{d}\left(\mathrm{M}-\mathrm{O}_{e q}\right)$ & $\mathrm{d}(\mathrm{Pt}-\mathrm{S})$ & $\mathrm{d}(\mathrm{M}-\mathrm{L})$ \\
\hline$\left[\mathrm{PtCo}(\mathrm{SAc})_{4}\left(\mathrm{OH}_{2}\right)\right]_{2}$ & 3.021 & 2.546 & 1.908 & 2.365 & 2.371 \\
& $(3.126)^{a}$ & $(2.634)$ & & & \\
{$\left[\mathrm{PtCo}(\mathrm{SAc})_{4}\left(3-\mathrm{NO}_{2} \mathrm{py}\right)\right]_{2}$} & 3.062 & 2.581 & 1.925 & 2.359 & 2.123 \\
& $(3.489)^{a}$ & $(2.634)$ & & & \\
{$\left[\mathrm{PtCo}\left(\mathrm{SAc}_{4}(\mathrm{py})\right]_{2}\right.$} & 3.062 & 2.592 & 1.928 & 2.358 & 2.146 \\
& & & & & \\
{$\left[\mathrm{PtCo}(\mathrm{tba})_{4}\left(\mathrm{OH}_{2}\right)\right]_{2}$} & 3.008 & 2.512 & 1.907 & 2.364 & 2.404 \\
& $(3.065)^{b}$ & $(2.599)$ & $(2.059)$ & $(2.342)$ & $(2.031)$ \\
{$\left[\mathrm{PtCo}(\mathrm{tba})_{4}\left(3-\mathrm{NO}_{2} \mathrm{py}\right)\right]_{2}$} & 3.050 & 2.541 & 1.924 & 2.359 & 2.136 \\
& & & & \\
{$\left[\mathrm{PtNi}\left(\mathrm{SAc}_{4}\left(\mathrm{OH}_{2}\right)\right]_{2}\right.$} & 3.080 & 2.571 & 2.320 & 2.315 & 2.067
\end{tabular}

Experimental data in parenthesis obtained from ${ }^{a}$ Inorg. Chem. 2013, 52, 4926-4933 and ${ }^{b}$ Chem. Sci. 2012, 3, 602-609. 
Table S3: Energy decomposition analysis in the Morokuyma-Ziegler scheme performed at different Pt-Pt distances for the $\left[\mathrm{PtCo}(\mathrm{SAc})_{4}\right]_{2}$ model complex in $\mathrm{C}_{2}$-point group. All values are in $\mathrm{eV}$.

\begin{tabular}{|c|c|c|c|c|c|c|c|}
\hline $\mathrm{d}(\mathrm{Pt}-\mathrm{Pt})$ & $\Delta V_{\text {elstat }}$ & $\Delta E_{\text {Pauli }}$ & $\Delta E_{o i-A}$ & $\Delta E_{o i-B}$ & $\Delta E_{o i}$ & $\Delta E_{\text {disp }}$ & $\Delta E_{i n t}$ \\
\hline 2.500 & -6.3665 & 11.0706 & -4.1883 & -0.5206 & -4.7089 & -1.4824 & -1.4863 \\
\hline 3.000 & -1.5573 & 2.7293 & -2.1212 & -0.1152 & -2.2364 & -1.1086 & -2.1630 \\
\hline 3.500 & -0.3456 & 0.6936 & -1.1743 & -0.0369 & -1.2112 & -0.7648 & -1.6281 \\
\hline 4.000 & -0.0539 & 0.1905 & -0.7073 & -0.0197 & -0.7270 & -0.4927 & -1.0831 \\
\hline 4.500 & 0.0111 & 0.0619 & -0.4660 & -0.0123 & -0.4783 & -0.3052 & -0.7105 \\
\hline 5.000 & 0.0223 & 0.0245 & -0.3334 & -0.0070 & -0.3404 & -0.1865 & -0.4800 \\
\hline 5.500 & 0.0218 & 0.0112 & -0.2565 & -0.0032 & -0.2597 & -0.1150 & -0.3417 \\
\hline 6.000 & 0.0193 & 0.0054 & -0.2097 & -0.0009 & -0.2106 & -0.0726 & -0.2585 \\
\hline
\end{tabular}



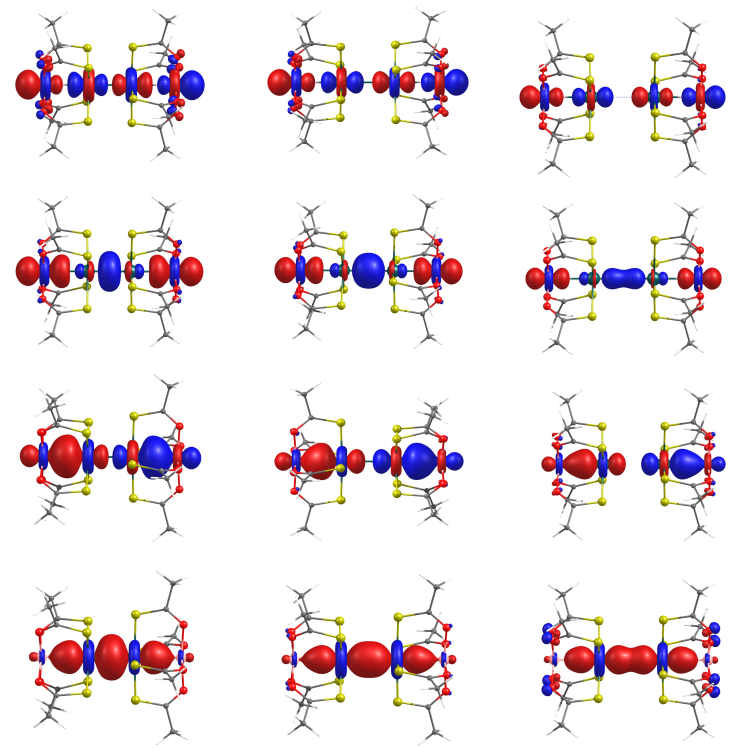

$2.500 \AA$

$3.000 \AA$

$3.500 \AA$
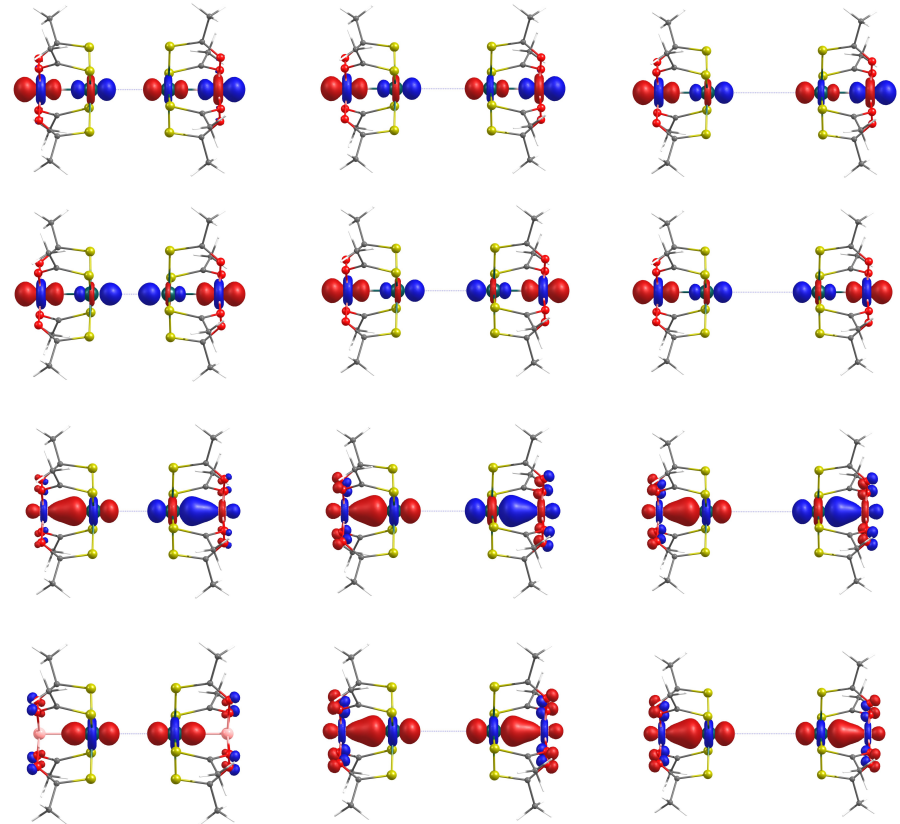

$4.000 \AA$

$5.000 \AA$

$6.000 \AA$

Figure S3: Representation of evolution at different Pt-Pt distances of the molecular orbitals belonging to A irreducible representation in bonding and anti-bonding combination. Calculations were performed in an idealized $\mathrm{C}_{2}$ point group structure. 

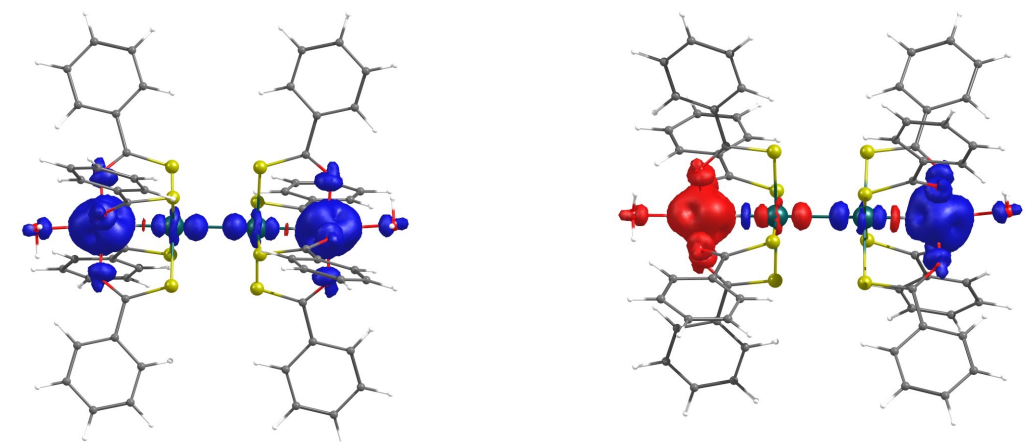

$\left[\mathrm{PtCo}(\mathrm{tba})_{4}\left(\mathrm{H}_{2} \mathrm{O}\right)\right]_{2}$
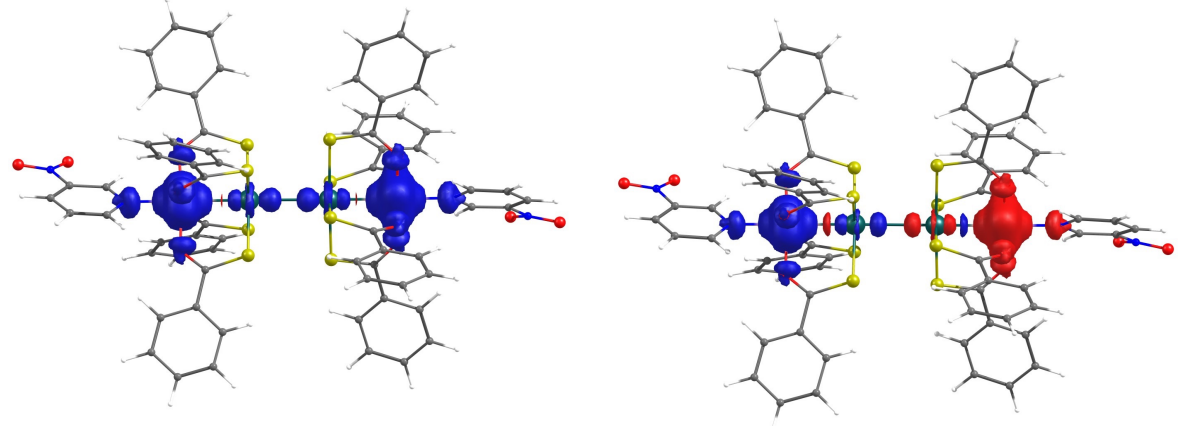

$\left[\mathrm{PtCo}(\mathrm{tba})_{4}\left(3-\mathrm{NO}_{2} \mathrm{py}\right)\right]_{2}$
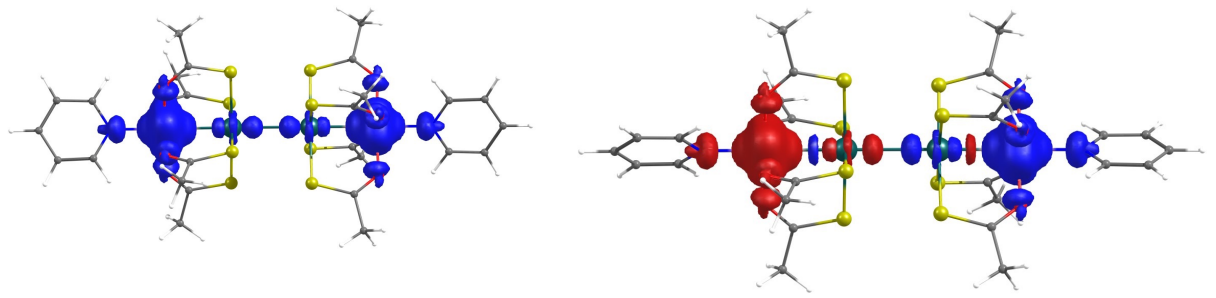

$\left[\mathrm{PtCo}(\mathrm{SAc})_{4}(\mathrm{py})\right]_{2}$

Figure S4: Spin densities isosurfaces for high spin (HS) (left) and broken-symmetry (BS) (right) states of $\left[P t C o(t b a)_{4}(Y)\right]_{2}, \mathrm{Y}$ $=\mathrm{H}_{2} \mathrm{O}$ and $3-\mathrm{NO}_{2}$ py. 
Table S4: Magnetic coupling constant calculations at broken-symmetry (BS) level considering different amounts of Hartree-Fock exchange in B3LYP functional. Experimental value $\mathbf{J}=\mathbf{- 1 2 . 7} \mathbf{c m}^{-1}{ }^{a}$

\begin{tabular}{|c|c|c|c|c|c|c|c|c|c|}
\hline \multicolumn{6}{|c|}{$\left[\mathrm{PtCo}(\mathrm{SAc})_{4}\left(\mathrm{H}_{2} \mathrm{O}\right)\right]_{2}$} & \multirow{2}{*}{$\begin{array}{c}\mathrm{HS}^{b} \\
\Delta \rho(\mathrm{Co})\end{array}$} & \multicolumn{3}{|c|}{$\mathrm{BS}$} \\
\hline$\% \mathrm{HF}$ & $J_{1}\left(\mathrm{~cm}^{-1}\right)$ & $J_{2}\left(\mathrm{~cm}^{-1}\right)$ & $J_{3}\left(\mathrm{~cm}^{-1}\right)$ & $<\hat{S}^{2}>_{H S}$ & $<\hat{S}^{2}>_{B S}$ & & $\Delta \rho(\mathrm{Pt})$ & $\Delta \rho(\mathrm{Co})$ & $\Delta \rho(\mathrm{Pt})$ \\
\hline 20 & -49.24 & -36.93 & -49.18 & 12.025 & 3.013 & 2.555 & 0.109 & 2.523 & 0.070 \\
\hline 25 & -40.63 & -30.47 & -40.61 & 12.023 & 3.018 & 2.587 & 0.099 & 2.559 & 0.064 \\
\hline 30 & -33.98 & -25.49 & -33.98 & 12.022 & 3.021 & 2.615 & 0.091 & 2.591 & 0.059 \\
\hline 35 & -28.78 & -21.59 & -28.79 & 12.021 & 3.022 & 6.639 & 0.084 & 2.619 & 0.055 \\
\hline 40 & -24.62 & -18.47 & -24.63 & 12.021 & 3.023 & 2.661 & 0.078 & 2.643 & 0.051 \\
\hline 45 & -21.25 & -15.94 & -21.26 & 12.020 & 3.024 & 2.681 & 0.073 & 2.664 & 0.048 \\
\hline 50 & -18.48 & -13.86 & -18.49 & 12.020 & 3.024 & 2.698 & 0.068 & 2.684 & 0.045 \\
\hline 55 & -16.18 & -12.14 & -16.19 & 12.019 & 2.023 & 2.714 & 0.064 & 2.701 & 0.043 \\
\hline 60 & -14.25 & -10.70 & -14.27 & 12.019 & 3.023 & 2.728 & 0.060 & 2.716 & 0.040 \\
\hline
\end{tabular}

${ }^{a}$ Experimental value reported in: Inorg. Chem. 2013, 52, 4926. DOI.10.1021/ic302531t.

${ }^{b}$ In these columns the spin-density over metal atoms in high-spin (HS) and broken-symmetry (BS) states are summarized. 
Table S5: Magnetic coupling constant calculations at broken-symmetry (BS) level considering different amounts of Hartree-Fock exchange in B3LYP functional. Experimental value $\mathbf{J}=-\mathbf{6 . 0} \mathbf{~ c m}^{-1}{ }^{a}$

\begin{tabular}{|c|c|c|c|c|c|c|c|c|c|}
\hline & & {$[P t C o(S A$} & ${ }_{4}\left(3-\mathrm{NO}_{2} \mathrm{~F}\right.$ & & & $\mathrm{HS}^{b}$ & & $\mathrm{BS}$ & \\
\hline$\% \mathrm{HF}$ & $J_{1}\left(\mathrm{~cm}^{-1}\right)$ & $J_{2}\left(\mathrm{~cm}^{-1}\right)$ & $J_{3}\left(\mathrm{~cm}^{-1}\right)$ & $<\hat{S}^{2}>_{H S}$ & $<\hat{S}^{2}>_{B S}$ & $\Delta \rho(\mathrm{Co})$ & $\Delta \rho(\mathrm{Pt})$ & $\Delta \rho(\mathrm{Co})$ & $\Delta \rho(\mathrm{Pt})$ \\
\hline 20 & -36.00 & -27.00 & -35.97 & 12.024 & 3.019 & 2.595 & 0.091 & 2.568 & 0.056 \\
\hline 25 & -29.54 & -22.16 & -29.54 & 12.022 & 3.021 & 2.625 & 0.082 & 2.603 & 0.051 \\
\hline 30 & -23.92 & -17.94 & -23.92 & 12.020 & 3.021 & 2.666 & 0.075 & 2.646 & 0.048 \\
\hline 35 & -20.14 & -15.10 & -20.14 & 12.020 & 3.022 & 2.698 & 0.069 & 2.672 & 0.044 \\
\hline 40 & -17.93 & -13.45 & -17.94 & 12.019 & 3.022 & 2.696 & 0.063 & 2.680 & 0.040 \\
\hline 45 & -15.55 & -11.67 & -15.56 & 12.019 & 3.022 & 2.714 & 0.059 & 2.698 & 0.037 \\
\hline 50 & -13.60 & -10.20 & -13.60 & 12.018 & 3.022 & 2.730 & 0.055 & 2.717 & 0.035 \\
\hline 55 & -11.92 & -8.94 & -11.93 & 12.018 & 3.021 & 2.744 & 0.051 & 2.732 & 0.033 \\
\hline 60 & -10.51 & -7.88 & -10.51 & 12.018 & 3.021 & 2.756 & 0.048 & 2.746 & 0.031 \\
\hline
\end{tabular}

${ }^{a}$ Experimental value reported in: Inorg. Chem. 2013, 52, 4926. DOI.10.1021/ic302531t.

${ }^{b}$ In these columns the spin-density over metal atoms in high-spin (HS) and broken-symmetry (BS) states are summarized. 
Table S6: Magnetic coupling constant calculations at broken-symmetry (BS) level considering different amounts of Hartree-Fock exchange in B3LYP functional. Experimental value $\mathbf{J}=\mathbf{- 1 0 . 0} \mathbf{~ c m}^{-1}{ }^{a}$

\begin{tabular}{|c|c|c|c|c|c|c|c|c|c|}
\hline \multicolumn{6}{|c|}{$\left[\mathrm{PtCo}(\mathrm{tba})_{4}\left(\mathrm{H}_{2} \mathrm{O}\right)\right]_{2}$} & \multirow{2}{*}{$\frac{\mathrm{HS}^{b}}{\Delta \rho(\mathrm{Co})}$} & \multicolumn{3}{|c|}{$\mathrm{BS}$} \\
\hline$\% \mathrm{HF}$ & $J_{1}\left(\mathrm{~cm}^{-1}\right)$ & $J_{2}\left(\mathrm{~cm}^{-1}\right)$ & $J_{2}\left(\mathrm{~cm}^{-1}\right)$ & $<\hat{S}^{2}>_{H S}$ & $<\hat{S}^{2}>_{B S}$ & & $\Delta \rho(\mathrm{Pt})$ & $\Delta \rho(\mathrm{Co})$ & $\Delta \rho(\mathrm{Pt})$ \\
\hline 20 & -43.76 & -32.82 & -43.72 & 12.022 & 3.013 & 2.605 & 0.102 & 2.574 & 0.067 \\
\hline 25 & -35.80 & -26.85 & -35.79 & 12.020 & 3.017 & 2.636 & 0.092 & 2.609 & 0.060 \\
\hline 30 & -29.73 & -22.30 & -29.73 & 12.019 & 3.019 & 2.663 & 0.084 & 2.640 & 0.055 \\
\hline 35 & -22.06 & -16.54 & -21.58 & 12.219 & 3.018 & 2.714 & 0.074 & 2.643 & 0.050 \\
\hline 40 & -21.15 & -15.86 & -21.16 & 12.018 & 3.021 & 2.707 & 0.071 & 2.690 & 0.047 \\
\hline 45 & -18.12 & -13.59 & -18.12 & 12.017 & 3.021 & 2.726 & 0.065 & 2.710 & 0.044 \\
\hline 50 & -15.62 & -11.71 & -15.62 & 12.017 & 3.021 & 2.742 & 0.061 & 2.728 & 0.041 \\
\hline 55 & -13.54 & -10.15 & -13.55 & 12.017 & 3.020 & 2.757 & 0.057 & 2.744 & 0.038 \\
\hline 60 & -14.95 & -11.21 & -14.95 & 12.017 & 3.021 & 2.754 & 0.054 & 2.742 & 0.034 \\
\hline
\end{tabular}

${ }^{a}$ Experimental value reported in: Chem. Sci. 2012, 3, 602. DOI.10.1039/c1sc00608h.

${ }^{b}$ In these columns the spin-density over metal atoms in high-spin (HS) and broken-symmetry (BS) states are summarized. 
Table S7: Magnetic coupling constant calculations at broken-symmetry (BS) level considering different amounts of Hartree-Fock exchange in B3LYP functional.

\begin{tabular}{|c|c|c|c|c|c|c|c|c|c|}
\hline & & {$[P t C o(t b a$} & $\left(3-\mathrm{NO}_{2} p\right.$ & & & $\mathrm{HS}^{a}$ & & BS & \\
\hline$\% \mathrm{HF}$ & $J_{1}\left(\mathrm{~cm}^{-1}\right)$ & $J_{2}\left(\mathrm{~cm}^{-1}\right)$ & $J_{2}\left(\mathrm{~cm}^{-1}\right)$ & $<\hat{S}^{2}>_{H S}$ & $<\hat{S}^{2}>_{B S}$ & $\Delta \rho(\mathrm{Co})$ & $\Delta \rho(\mathrm{Pt})$ & $\Delta \rho(\mathrm{Co})$ & $\Delta \rho(\mathrm{Pt})$ \\
\hline 20 & -36.27 & -27.11 & -36.12 & 12.027 & 3.020 & 2.604 & 0.094 & 2.579 & 0.060 \\
\hline 25 & -29.88 & -22.41 & -29.87 & 12.024 & 3.022 & 2.635 & 0.085 & 2.613 & 0.055 \\
\hline 30 & -25.02 & -18.76 & -25.02 & 12.023 & 3.023 & 2.661 & 0.078 & 2.642 & 0.051 \\
\hline 35 & -21.86 & -16.40 & -21.86 & 12.021 & 3.023 & 2.671 & 0.071 & 2.654 & 0.045 \\
\hline 40 & -18.89 & -14.17 & -18.89 & 12.020 & 3.023 & 2.692 & 0.065 & 2.676 & 0.042 \\
\hline 45 & -16.44 & -12.33 & -16.44 & 12.020 & 3.023 & 2.710 & 0.061 & 2.696 & 0.039 \\
\hline 50 & -14.38 & -10.78 & -14.39 & 12.019 & 3.023 & 2.726 & 0.057 & 2.714 & 0.036 \\
\hline 55 & -12.64 & -9.48 & -12.64 & 12.019 & 3.022 & 2.740 & 0.053 & 2.729 & 0.034 \\
\hline 60 & -11.17 & -8.38 & -11.17 & 12.019 & 3.022 & 2.753 & 0.050 & 2.743 & 0.032 \\
\hline
\end{tabular}

${ }^{a}$ In these columns the spin-density over metal atoms in high-spin (HS) and broken-symmetry (BS) states are summarized. 
Table S8: Magnetic coupling constant calculations at broken-symmetry (BS) level considering different amounts of Hartree-Fock exchange in B3LYP functional.

\begin{tabular}{|c|c|c|c|c|c|c|c|c|c|}
\hline \multicolumn{6}{|c|}{$\left[\mathrm{PtCo}(S A c)_{4}(p y)\right]_{2}$} & \multirow{2}{*}{$\begin{array}{c}\mathrm{HS}^{a} \\
\Delta \rho(\mathrm{Co})\end{array}$} & \multicolumn{3}{|c|}{$\mathrm{BS}$} \\
\hline$\% \mathrm{HF}$ & $J_{1}\left(\mathrm{~cm}^{-1}\right)$ & $J_{2}\left(\mathrm{~cm}^{-1}\right)$ & $J_{3}\left(\mathrm{~cm}^{-1}\right)$ & $<\hat{S}^{2}>_{H S}$ & $<\hat{S}^{2}>_{B S}$ & & $\Delta \rho(\mathrm{Pt})$ & $\Delta \rho(\mathrm{Co})$ & $\Delta \rho(\mathrm{Pt})$ \\
\hline 20 & -34.09 & -25.57 & -34.07 & 12.023 & 3.017 & 2.616 & 0.089 & 2.589 & 0.056 \\
\hline 25 & -28.71 & -21.53 & -28.70 & 12.020 & 3.019 & 2.628 & 0.080 & 2.606 & 0.050 \\
\hline 30 & -23.95 & -17.97 & -23.96 & 12.019 & 3.021 & 2.655 & 0.073 & 2.636 & 0.045 \\
\hline 35 & -20.29 & -15.22 & -20.30 & 12.019 & 3.021 & 2.678 & 0.067 & 2.661 & 0.041 \\
\hline 40 & -17.41 & -13.06 & -17.42 & 12.019 & 3.022 & 2.700 & 0.062 & 2.683 & 0.0385 \\
\hline 45 & -15.05 & -11.29 & -15.06 & 12.018 & 3.022 & 2.717 & 0.058 & 2.703 & 0.036 \\
\hline 50 & -13.17 & -9.88 & -13.18 & 12.018 & 3.021 & 2.733 & 0.054 & 2.720 & 0.034 \\
\hline 55 & -11.54 & -8.66 & -11.55 & 12.018 & 3.021 & 2.747 & 0.050 & 2.735 & 0.032 \\
\hline 60 & -10.26 & -7.70 & -10.27 & 12.018 & 3.021 & 2.759 & 0.047 & 2.749 & 0.030 \\
\hline
\end{tabular}

${ }^{a}$ In these columns the spin-density over metal atoms in high-spin (HS) and broken-symmetry (BS) states are summarized.

Table S9: Magnetic coupling constant calculations at broken-symmetry (BS) level considering different amounts of Hartree-Fock exchange in B3LYP functional. Experimental value $\mathbf{J}=\mathbf{- 6 . 0} \mathbf{c m}^{-1}{ }^{a}$

\begin{tabular}{|c|c|c|c|c|c|c|c|c|c|}
\hline \multicolumn{6}{|c|}{$\left[\mathrm{PtNi}(\mathrm{SAc})_{4}\left(\mathrm{H}_{2} \mathrm{O}\right)\right]_{2}$} & \multirow{2}{*}{$\frac{\mathrm{HS}^{a}}{\Delta \rho(\mathrm{Ni})}$} & \multicolumn{3}{|c|}{$\mathrm{BS}$} \\
\hline$\% \mathrm{HF}$ & $J_{1}\left(\mathrm{~cm}^{-1}\right)$ & $J_{2}\left(\mathrm{~cm}^{-1}\right)$ & $J_{3}\left(\mathrm{~cm}^{-1}\right)$ & $<\hat{S}^{2}>_{H S}$ & $<\hat{S}^{2}>_{B S}$ & & $\Delta \rho(\mathrm{Pt})$ & $\Delta \rho(\mathrm{Ni})$ & $\Delta \rho(\mathrm{Pt})$ \\
\hline 20 & -90.39 & -60.26 & -90.20 & 6.012 & 2.004 & 1.653 & 0.094 & -1.616 & -0.070 \\
\hline 25 & -71.47 & -47.65 & -71.43 & 6.012 & 2.010 & 1.685 & 0.084 & -1.658 & -0.063 \\
\hline 30 & -57.42 & -38.28 & -57.43 & 6.012 & 2.013 & 1.713 & 0.076 & -1.687 & -0.057 \\
\hline 35 & -46.82 & -31.21 & -46.85 & 6.012 & 2.015 & 1.737 & 0.069 & -1.715 & -0.052 \\
\hline 40 & -38.79 & -25.86 & -38.83 & 6.012 & 2.016 & 1.758 & 0.062 & -1.740 & -0.047 \\
\hline 45 & -32.50 & -21.67 & -32.53 & 6.012 & 2.016 & 1.776 & 0.057 & -1.760 & -0.042 \\
\hline
\end{tabular}

${ }^{a}$ Experimental value reported in: Inorg. Chem. 2013, 52, 4926. DOI.10.1021/ic302531t.

${ }^{b}$ In these columns the spin-density over metal atoms in high-spin (HS) and broken-symmetry (BS) states are summarized. 
Cartesian coordinates of the optimized complexes

\begin{tabular}{|c|c|c|c|}
\hline \multicolumn{4}{|c|}{$\left[\mathrm{PtCo}(\mathrm{SAc})_{4}\left(\mathrm{H}_{2} \mathrm{O}\right)\right]_{2}$} \\
\hline Co & 864000 & -0.000034000 & 4.051792000 \\
\hline Co & 0.001845000 & 0.004652000 & -4.052539000 \\
\hline $\mathrm{Pt}$ & -0.003660000 & -0.000632000 & 1.505767000 \\
\hline $\mathrm{Pt}$ & -0.002287000 & -0.000512000 & -1.506702000 \\
\hline $\mathrm{S}$ & 2.359259000 & -0.036363000 & 1.563199000 \\
\hline $\mathrm{s}$ & -0.029974000 & -2.363472000 & 1.564455000 \\
\hline $\mathrm{s}$ & -2.368858000 & 0.026703000 & 1.564015000 \\
\hline $\mathrm{s}$ & 0.032193000 & 2.364680000 & 1.561108000 \\
\hline $\mathrm{s}$ & -1.708990000 & -1.634976000 & -1.565922000 \\
\hline $\mathrm{S}$ & -1.638797000 & 1.707875000 & -1.559526000 \\
\hline $\mathrm{s}$ & 1.712336000 & 1.629351000 & -1.557619000 \\
\hline $\mathrm{s}$ & 1.625276000 & -1.713774000 & -1.564347000 \\
\hline $\mathrm{O}$ & 1.860279000 & -0.371601000 & 4.162290000 \\
\hline $\mathrm{O}$ & -0.388547000 & -1.863417000 & 4.159831000 \\
\hline $\mathrm{O}$ & -1.884857000 & 0.380176000 & 4.158596000 \\
\hline $\mathrm{O}$ & 0.368967000 & 1.881471000 & 4.158238000 \\
\hline $\mathrm{O}$ & -1.122778000 & -1.529569000 & -4.166394000 \\
\hline $\mathrm{O}$ & -1.544736000 & 1.139416000 & -4.159457000 \\
\hline $\mathrm{O}$ & 1.132014000 & 1.554000000 & -4.155262000 \\
\hline $\mathrm{O}$ & 1.542538000 & -1.110782000 & -4.161401000 \\
\hline $\mathrm{O}$ & -0.163112000 & 0.238765000 & 6.407780000 \\
\hline $\mathrm{H}$ & -1.128822000 & 0.355143000 & 6.325007000 \\
\hline $\mathrm{H}$ & 0.189800000 & 1.146640000 & 6.347876000 \\
\hline $\mathrm{O}$ & -0.040757000 & 0.295598000 & -6.405574000 \\
\hline $\mathrm{H}$ & 0.611567000 & 1.017545000 & -6.327266000 \\
\hline $\mathrm{H}$ & -0.902703000 & 0.748278000 & -6.336734000 \\
\hline C & 2.702626000 & -0.312073000 & 3.220005000 \\
\hline $\mathrm{C}$ & 4.152877000 & -0.521402000 & 3.589139000 \\
\hline $\mathrm{H}$ & 4.426423000 & 0.166495000 & 4.401986000 \\
\hline $\mathrm{H}$ & 4.816870000 & -0.367262000 & 2.731165000 \\
\hline $\mathrm{H}$ & 4.281586000 & -1.547711000 & 3.964704000 \\
\hline $\mathrm{C}$ & -0.317951000 & -2.706656000 & 3.219236000 \\
\hline C & -0.522447000 & -4.157380000 & 3.588710000 \\
\hline $\mathrm{H}$ & -1.548215000 & -4.291056000 & 3.964085000 \\
\hline $\mathrm{H}$ & 0.167144000 & -4.426509000 & 4.401558000 \\
\hline $\mathrm{H}$ & -0.365084000 & -4.821010000 & 2.731033000 \\
\hline $\mathrm{C}$ & -2.727270000 & 0.306166000 & 3.211546000 \\
\hline C & -4.181103000 & 0.495560000 & 3.575503000 \\
\hline $\mathrm{H}$ & -4.459475000 & -0.226070000 & 4.357478000 \\
\hline $\mathrm{H}$ & -4.836860000 & 0.368507000 & 2.707077000 \\
\hline $\mathrm{H}$ & -4.321350000 & 1.506139000 & 3.988179000 \\
\hline C & 0.305741000 & 2.722790000 & 3.209791000 \\
\hline $\mathrm{C}$ & 0.505978000 & 4.175750000 & 3.572265000 \\
\hline $\mathrm{H}$ & 1.522253000 & 4.309764000 & 3.972838000 \\
\hline $\mathrm{H}$ & -0.204343000 & 4.458755000 & 4.362862000 \\
\hline $\mathrm{H}$ & 0.373083000 & 4.832436000 & 2.705397000 \\
\hline C & -1.778676000 & -2.062423000 & -3.224698000 \\
\hline C & -2.701320000 & -3.199799000 & -3.596696000 \\
\hline $\mathrm{H}$ & -3.376554000 & -2.875109000 & -4.401393000 \\
\hline $\mathrm{H}$ & -3.285911000 & -3.546636000 & -2.737244000 \\
\hline $\mathrm{H}$ & -2.099769000 & -4.035319000 & -3.985129000 \\
\hline $\mathrm{C}$ & -2.074392000 & 1.794636000 & -3.209896000 \\
\hline C & -3.206911000 & 2.726367000 & -3.572900000 \\
\hline $\mathrm{H}$ & -4.040594000 & 2.136123000 & -3.982513000 \\
\hline $\mathrm{H}$ & -2.874128000 & 3.421921000 & -4.357276000 \\
\hline $\mathrm{H}$ & -3.561014000 & 3.292713000 & -2.704483000 \\
\hline $\mathrm{C}$ & 1.795484000 & 2.073688000 & -3.205706000 \\
\hline C & 2.735600000 & 3.199646000 & -3.566913000 \\
\hline $\mathrm{H}$ & 3.433853000 & 2.858429000 & -4.345248000 \\
\hline $\mathrm{H}$ & 3.298977000 & 3.553199000 & -2.696345000 \\
\hline $\mathrm{H}$ & 2.153833000 & 4.035750000 & -3.983685000 \\
\hline $\mathrm{C}$ & 2.065287000 & -1.775284000 & -3.220078000 \\
\hline C & 3.199519000 & -2.702536000 & -3.589729000 \\
\hline $\mathrm{H}$ & 2.871198000 & -3.376955000 & -4.393629000 \\
\hline $\mathrm{H}$ & 3.542683000 & -3.287860000 & -2.729305000 \\
\hline $\mathrm{H}$ & 4.038560000 & -2.105955000 & -3.978288000 \\
\hline
\end{tabular}


$\left[\mathrm{PtCo}(\mathrm{SAc})_{4}\left(\mathrm{NO}_{2} \mathrm{py}\right)\right]_{2}$

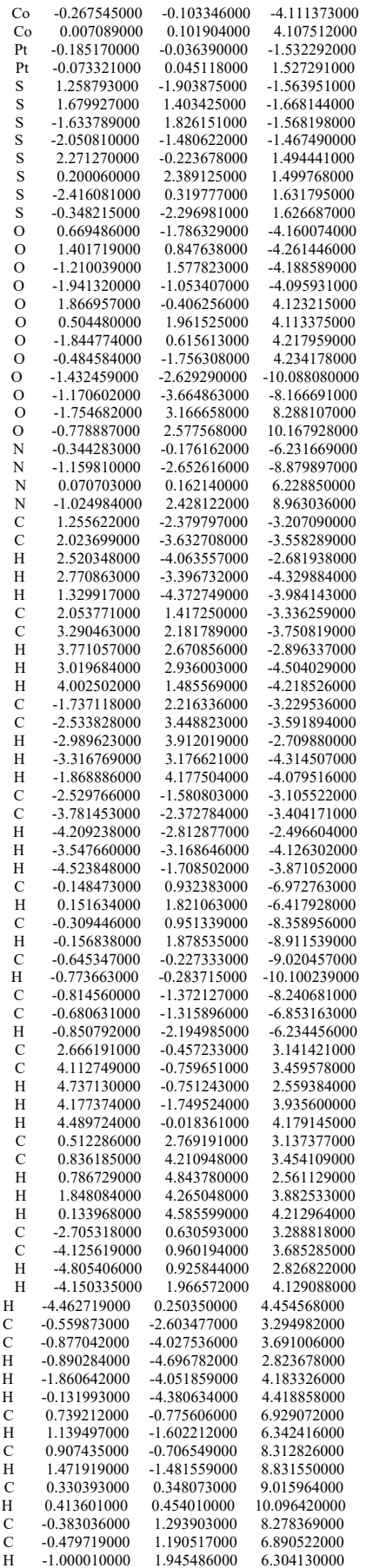


$\left[\operatorname{PtCo}(\mathrm{SAc})_{4}(\mathrm{py})\right]_{2}$

\begin{tabular}{|c|c|c|}
\hline 19029 & 01 & 3 \\
\hline 0.020052113 & -0.004034447 & -4.122469632 \\
\hline 0.000000000 & 0.000000000 & 1.530896552 \\
\hline 0.000000000 & 0.000000000 & -1.530896552 \\
\hline 2.358478439 & 0.000000000 & 1.560296052 \\
\hline 0.003785205 & -2.358317569 & 1.559112283 \\
\hline-2.357559787 & 0.001893925 & 1.575607267 \\
\hline-0.006049554 & 2.357481998 & 1.571002366 \\
\hline 1.664271973 & -1.672375265 & -1.556469042 \\
\hline-1.666604057 & -1.667791531 & -1.574643106 \\
\hline-1.664385217 & 1.669305507 & -1.580639743 \\
\hline 1.664975250 & 1.669732024 & -1.556711935 \\
\hline 1.916123831 & 0.277935533 & 4.175262471 \\
\hline 0.281112713 & -1.916279409 & 4.175021166 \\
\hline-1.894770999 & -0.292862565 & 4.185775106 \\
\hline-0.258724282 & 1.901417995 & 4.187283790 \\
\hline 1.569325404 & -1.148315160 & -4.169732039 \\
\hline-1.137825808 & -1.544993305 & -4.186617027 \\
\hline-1.527320901 & 1.143866366 & -4.191713533 \\
\hline 1.180495486 & 1.535368629 & -4.176734642 \\
\hline 0.019460493 & -0.014292548 & 6.267013351 \\
\hline 0.033786380 & -0.006392990 & -6.268115627 \\
\hline 2.737623884 & 0.220582244 & 3.213281204 \\
\hline 4.201959992 & 0.372028011 & 3.562028591 \\
\hline 4.842896830 & 0.248230168 & 2.681936974 \\
\hline 4.473527406 & -0.370632570 & 4.326278016 \\
\hline 4.368880488 & 1.371067031 & 3.992103641 \\
\hline 0.226756685 & -2.736838585 & 3.211958790 \\
\hline 0.382750729 & -4.200975193 & 3.559931990 \\
\hline 0.292978455 & -4.840265761 & 2.674544368 \\
\hline-0.380496963 & -4.485236511 & 4.299147098 \\
\hline 1.368664566 & -4.357153921 & 4.022653573 \\
\hline-2.723337156 & -0.229719019 & 3.230349287 \\
\hline-4.184767552 & -0.384359957 & 3.589396580 \\
\hline-4.833217649 & -0.249805529 & 2.716380063 \\
\hline-4.449972668 & 0.348162117 & 4.365508571 \\
\hline-4.348142032 & -1.388999260 & 4.007759879 \\
\hline-0.215669363 & 2.726732887 & 3.227770077 \\
\hline-0.376678949 & 4.188407763 & 3.583749200 \\
\hline-0.272087595 & 4.833943151 & 2.704553481 \\
\hline 0.372287307 & 4.467320157 & 4.339311651 \\
\hline-1.371192975 & 4.341268549 & 4.029062439 \\
\hline-0.687779608 & -0.926578250 & 6.957464246 \\
\hline-1.255387297 & -1.636731473 & 6.355184335 \\
\hline-0.705027611 & -0.970102021 & 8.351041418 \\
\hline-1.295790509 & -1.730681601 & 8.862956906 \\
\hline 0.041183749 & -0.030341436 & 9.064452256 \\
\hline 0.049636826 & -0.036568794 & 10.155586638 \\
\hline 0.776167555 & 0.917825659 & 8.350329146 \\
\hline 0.737651918 & 0.889913675 & 6.956874742 \\
\hline 1.295426964 & 1.607580685 & 6.354299551 \\
\hline 2.100644233 & -1.775704531 & -3.206552186 \\
\hline 3.243400915 & -2.705252524 & -3.551495318 \\
\hline 3.596869092 & -3.256552028 & -2.672981708 \\
\hline 4.075622628 & -2.115953860 & -3.965371723 \\
\hline 2.916895377 & -3.412220099 & -4.327930107 \\
\hline-1.762870924 & -2.089582841 & -3.229402060 \\
\hline-2.683160961 & -3.236032122 & -3.587070846 \\
\hline-3.217785561 & -3.616760864 & -2.709636228 \\
\hline-2.087867248 & -4.048629826 & $\quad-4.029737669$ \\
\hline-3.406525094 & -2.902933035 & -4.345628440 \\
\hline-2.072858194 & 1.773437536 & -3.237959385 \\
\hline-3.206319877 & 2.706835822 & -3.602756718 \\
\hline-3.579366554 & 3.253172173 & -2.729266058 \\
\hline-4.028980416 & 2.121597535 & -4.040636074 \\
\hline-2.860098783 & 3.418424173 & -4.366345200 \\
\hline 1.789857376 & 2.083535403 & -3.211510048 \\
\hline 2.716631951 & 3.228404561 & -3.557754955 \\
\hline 3.233561922 & 3.614021843 & -2.671918061 \\
\hline 2.130330548 & 4.038457981 & -4.016874428 \\
\hline 3.455225276 & 2.891096399 & -4.299566736 \\
\hline 1.185259140 & 0.121024954 & -6.951511531 \\
\hline 2.085623537 & 0.218423201 & -6.343989061 \\
\hline 1.238310747 & 0.126292384 & -8.344824115 \\
\hline 2.198 & 0.232453278 & -8.850959928 \\
\hline 0.050062814 & -0.006658108 & -9.065531777 \\
\hline 0.056457392 & -0.006754418 & -10.156716432 \\
\hline-1.14 & -0.13 & -8.358 \\
\hline-1.109624894 & -0.133773892 & -6.964972213 \\
\hline-2.017116250 & -0.231105992 & -6.368065038 \\
\hline & & \\
\hline $1.9 / 40 / 0 / 28$ & 1.672594873 & 8.861822349 \\
\hline
\end{tabular}


$\left[\mathrm{PtNi}(\mathrm{SAc})_{4}\left(\mathrm{H}_{2} \mathrm{O}\right)\right]_{2}$

\begin{tabular}{|c|c|c|c|}
\hline & J & & \\
\hline & 0.066688000 & -0.005434000 & 00780 \\
\hline & -0.025997000 & 0.000000000 & -1.539468000 \\
\hline & 0.025997000 & 0.000000000 & 1.5394680 \\
\hline & 1.395350000 & -1.825264000 & -1.561381000 \\
\hline & 1.810068000 & 1.416458000 & -1.61134900 \\
\hline & -1.414266000 & 1.858619000 & -1.555121000 \\
\hline & -1.865365000 & -1.413113000 & 447280 \\
\hline & 0.008982000 & 2.329015000 & 1.5567180 \\
\hline & -2.289432000 & 0.000000000 & 1.5394680 \\
\hline & -0.019718000 & -2.325895000 & \\
\hline & 2.345362000 & 0.03 & \\
\hline o & 1.278226000 & -1.487862000 & -4.1668710 \\
\hline & 1.32 & & \\
\hline $\mathrm{O}$ & -1.525331000 & & \\
\hline $\mathrm{O}$ & -1.594046000 & $\begin{array}{r}-1.444262000 \\
\end{array}$ & -4.0 \\
\hline & & & \\
\hline & -1.91 & & \\
\hline o & -0.327774000 & -1.975465000 & 4.16 \\
\hline & & & \\
\hline & -0.16 & & \\
\hline $\mathrm{H}$ & 09000 & 371000 & -6.4 \\
\hline & & & \\
\hline & & & \\
\hline $\mathrm{H}$ & 52000 & 0.1794 & 000 \\
\hline & & & \\
\hline & & & \\
\hline & 2.805777000 & -3.15 & -3.4 \\
\hline $\mathrm{H}$ & 2.524494000 & & \\
\hline & 2.89 & & \\
\hline n & 3.666400000 & -2.72 & -3.6 \\
\hline & 47000 & & \\
\hline & & & \\
\hline $\mathrm{H}$ & 2.86 & 00 & -4.3 \\
\hline$\Pi$ & & & \\
\hline & & & \\
\hline $\mathrm{C}$ & -1.90 & 00 & \\
\hline & & & \\
\hline & & & \\
\hline & & & \\
\hline & & & \\
\hline & & & \\
\hline & -3.37 & -2.7 & \\
\hline $\mathrm{H}$ & -3 & & \\
\hline $\mathrm{H}$ & -4.1 & -2.2 & \\
\hline & -3.607 & -3.157 & \\
\hline & & & \\
\hline & & & \\
\hline & & & \\
\hline & & & \\
\hline & & & \\
\hline & & & \\
\hline & & & \\
\hline & & & \\
\hline & & & \\
\hline & & & \\
\hline & & & \\
\hline & & & \\
\hline & & & \\
\hline & & & \\
\hline & & & \\
\hline & & -0.239 & \\
\hline & & & \\
\hline & & & \\
\hline & 814150 & 00 & \\
\hline & 562 & -1.125072000 & 7483046 \\
\hline
\end{tabular}


$\left[\mathrm{PtCo}(\mathrm{tba})_{4}\left(\mathrm{H}_{2} \mathrm{O}\right)\right]_{2}$

$\begin{array}{llll}\mathrm{Pt} & 8.486322000 & 1.043643000 & 11.283137000\end{array}$

$\begin{array}{llll} & 8.574190000 & -1.057286000 & 11.274611000\end{array}$

$\begin{array}{lrrr}\text { Co } & 6.718089000 & 2.822843000 & 11.329721000\end{array}$

$\begin{array}{ccc}12.342817000 & -2.836476000 & 11.306001000 \\ 9.206801000 & 1.810822000 & 13.415663000\end{array}$

$\begin{array}{lll}9.937727000 & 2.597233000 & 10.224792000\end{array}$

$\begin{array}{lll}6.887822000 & 3.127484000 & 13.234421000 \\ 7.972933000 & 4.235846000 & 11.008898000\end{array}$

$\begin{array}{lll}7.972933000 & 4.235846000 & 11.008898000 \\ 4.852189000 & 4.410261000 & 11.665370000\end{array}$

$\begin{array}{lll}4.852189000 & 4.410261000 & 11.665370000 \\ 4.250915000 & 3.640705000 & 11.624179000\end{array}$

$\begin{array}{lll}4.250915000 & 3.640705000 & 11.624179000 \\ 7.895208000 & 2.798780000 & 13.949849000\end{array}$

$\begin{array}{lll}7.895208000 & 2.798780000 & 13.949849000 \\ 7.951831000 & 3.332000000 & 15.339245000\end{array}$

$\begin{array}{lll}7.9583600 & 3.33206000 & 15.339245000 \\ 7.193946000 & 4.470265000 & 15.670613000\end{array}$

$\begin{array}{lll}7.193946000 & 4.470265000 & 15.670613000 \\ 6.585094000 & 4.943285000 & 14.901592000\end{array}$

$\begin{array}{lll}7.58084000 & 4.943285000 & 14.901592000 \\ 7.240802000 & 4.999639000 & 16.959176000\end{array}$

$\begin{array}{lll}7.240802000 & 4.9986311000 & 16.959176000 \\ 6.654832000 & 5.8873110268000\end{array}$

$\begin{array}{llll}8.041751000 & 4.399706000 & 17.936347000\end{array}$

$\begin{array}{llll}8.078706000 & 4.815506000 & 18.944537000\end{array}$

$\begin{array}{lll}9.135526000 & 4.107088000 & 10.501748000\end{array}$

$\begin{array}{lll}9.837884000 & 5.360141000 & 10.107643000\end{array}$

$\begin{array}{lll}9.095440000 & 6.552558000 & 10.019772000\end{array}$

$\begin{array}{llll}8.029099000 & 6.523995000 & 10.240432000\end{array}$

$\begin{array}{llll}9.718049000 & 7.742343000 & 9.646246000\end{array}$

$\begin{array}{llll}9.133151000 & 8.660792000 & 9.578237000\end{array}$

$\begin{array}{lll}11.086106000 & 7.759254000 & 9.355176000\end{array}$

$\begin{array}{llll}11.572051000 & 8.691210000 & 9.062162000\end{array}$

$\begin{array}{llll}5.132274000 & 4.418277000 & 12.602751000\end{array}$

$\begin{array}{llll}9.847504000 & -1.848732000 & 13.396349000\end{array}$

$\begin{array}{lll}9.125422000 & -2.599848000 & 10.196607000\end{array}$

$\begin{array}{lll}12.167033000 & -3.163544000 & 13.206392000 \\ 11.089479000 & -4.246211000 & 10.96602000\end{array}$

$\begin{array}{lll}11.089479000 & -4.246211000 & 10.966061000\end{array}$

$\begin{array}{rrr}14.212231000 & -4.424959000 & 11.629029000\end{array}$

$\begin{array}{llll}14.810456000 & -3.652634000 & 11.595809000 \\ 11.156452000 & -2.84485000 & 13.921833000\end{array}$

$\begin{array}{lll}11.156452000 & -2.844865000 & 13.921833000 \\ 11.092345000 & -3.39939600 & 15.30252000\end{array}$

$\begin{array}{lll}11.092345000 & -3.399396000 & 15.302526000 \\ 11.845404000 & -4545036000 & 15.619114000\end{array}$

$\begin{array}{lll}11.845404000 & -4.545036000 & 15.619114000 \\ 12.456799000 & -5.007206000 & 14.845545000\end{array}$

$\begin{array}{lll}11.456799000 & -5.007206000 & 14.845545000 \\ 11.790011000 & -5.095496000 & 16.898451000\end{array}$

$\begin{array}{lll}11.790011000 & -5.095496000 & 16.898451000 \\ 12.372219000 & -5.988757000 & 17.130006000\end{array}$

$\begin{array}{lll}12.372219000 & -5.988757000 & 17.130006000 \\ 10.985267000 & -4.509707000 & 17.880999000\end{array}$

$\begin{array}{llll}10.985267000 & -4.504707000 & 17.880999000 \\ 10.941472000 & -4.942262000 & 18.881790000\end{array}$

$\begin{array}{llll}10.941472000 & -4.942262000 & 18.881790000 \\ 10.236095000 & -3.368387000 & 17.576254000\end{array}$

$\begin{array}{llll}10.23645000 & -3.368387000 & 17.576254000 \\ 9.611684000 & -2.903678000 & 18.340362000\end{array}$

$\begin{array}{rrr}9.61168400 & -3.368387000 & 17.57625000 \\ 10.288943000 & -2.814552000 & 18.340362000 \\ 9.71598298186000\end{array}$

\begin{tabular}{rrr}
10.288943000 & -2.814552000 & 16.298186000 \\
9.715948000 & -1.917573000 & 16.062147000 \\
\hline .927354000 & -4.11243000 & 10.459122000
\end{tabular}

\begin{tabular}{lll}
9.927354000 & -4.112423000 & 10.062147000 \\
\hline
\end{tabular}

$\begin{array}{lll}9.927354000 & -4.112423000 & 10.459122000 \\ 9.225338000 & -5.361670000 & 10.052535000\end{array}$

$\begin{array}{rrr}9.225338000 & -5.361670000 & 10.052535000 \\ 9.967523000 & -6.553575000 & 9.955942000\end{array}$

$\begin{array}{llll}\text { H } & 11.033523000 & -6.527228000 & 10.178516000\end{array}$

$\begin{array}{lrrr}\text { C } \quad 9.345083000 & -7.740074000 & 9.571892000\end{array}$ 


\begin{tabular}{|c|c|c|c|}
\hline & & & \\
\hline & 7.977461000 & -7.754176000 & 9.278630000 \\
\hline & 7.491624000 & -8.683592000 & 8.977530000 \\
\hline & 7.233298000 & -6.573211000 & 9.373041000 \\
\hline & 6.165350000 & -6.580257000 & 9.150273000 \\
\hline & 7.849966000 & -5.384362000 & 9.759991000 \\
\hline & 267526000 & -4.466727000 & 9.847620000 \\
\hline & 13.930380000 & -4.442365000 & 12.565742000 \\
\hline & 11.385038000 & -0.365969000 & 9.147982000 \\
\hline & 12.115310000 & 0.405853000 & 12.335059000 \\
\hline & 12.686318000 & -2.687606000 & 9.426732000 \\
\hline & 13.772148000 & -1.577432000 & 11.660135000 \\
\hline & 12.384282000 & -1.700343000 & 8.678611000 \\
\hline & 12.961180000 & -1.701029000 & 7.304627000 \\
\hline & 14.016621000 & -2.586149000 & 7.016113000 \\
\hline $\mathrm{H}$ & 14.374638000 & -3.249387000 & 7.802435000 \\
\hline & 14.592254000 & -2.605297000 & 5.747166000 \\
\hline & 15.412437000 & -3.292935000 & 5.535181000 \\
\hline C & 14.12 & & 4.748879000 \\
\hline $\mathrm{H}$ & 14.573763000 & & 000 \\
\hline & 13.068834000 & -0.867718000 & 5.026248000 \\
\hline & & & \\
\hline & & & \\
\hline & 11.659018000 & 97000 & 12000 \\
\hline & 13.6 & & \\
\hline & 14.8 & & \\
\hline & 16.081108000 & 00000 & 12.6 \\
\hline & 16.0 & & 52000 \\
\hline & 17.2 & & \\
\hline & 18.184980000 & -0.28 & 28000 \\
\hline & 17 & & 41000 \\
\hline H & 18.1 & & \\
\hline & 16.032565000 & 9000 & 30000 \\
\hline & 16.0 & & 91000 \\
\hline C & 14.8 & & \\
\hline & 13.92 & 1000 & 346000 \\
\hline & & & \\
\hline s & 6.94 & -0.4 & \\
\hline o & 92000 & 2.6 & 67000 \\
\hline & & & \\
\hline C & & & \\
\hline & 6.115 & 00 & 2000 \\
\hline & & & \\
\hline $\mathrm{H}$ & & & \\
\hline & 4.494 & 00 & 0000 \\
\hline & & & \\
\hline C & & & \\
\hline & 4.52 & & 000 \\
\hline C & & & \\
\hline $\mathrm{H}$ & & & \\
\hline & & & \\
\hline $\mathrm{H}$ & & & \\
\hline C & & & \\
\hline C & & & \\
\hline C & & & \\
\hline $\mathrm{H}$ & & & \\
\hline C & 1.80 & & \\
\hline $\mathrm{H}$ & & & \\
\hline C & & & \\
\hline $\mathrm{H}$ & & & \\
\hline C & & & \\
\hline $\mathrm{H}$ & & & \\
\hline C & & & \\
\hline $\mathrm{H}$ & & & \\
\hline C & & & \\
\hline $\mathrm{H}$ & & & \\
\hline $\mathrm{C}$ & & & \\
\hline $\mathrm{H}$ & & & \\
\hline C & & & \\
\hline & & & \\
\hline & & & \\
\hline & & 46756 & 808 \\
\hline
\end{tabular}


$\left[\mathrm{PtCo}(\mathrm{tba})_{4}\left(\mathrm{NO}_{2} \mathrm{py}\right)\right]_{2}$

$\begin{array}{llll}\text { Pt } & 10.261102000 & -0.455493000 & 11.605806000\end{array}$

$\begin{array}{llll}\text { Co } & 12.052915000 & -2.250575000 & 11.632596000\end{array}$

$\begin{array}{cccc}\mathrm{S} & 10.034786000 & -0.700879000 & 13.957753000 \\ \mathrm{~S} & 8.621267000 & -2.140934000 & 11.261641000\end{array}$

$\begin{array}{rrr}8.621267000 & -2.140934000 & 11.261641000 \\ 11.514725000 & -2.858126000 & 13.397045000\end{array}$

$\begin{array}{lll}11.514725000 & -2.858126000 & 13.397045000 \\ 10.850167000 & -3.547424000 & 10.826465000\end{array}$

$\begin{array}{lll}10.821018000 & -2.210071000 & 14.248748000\end{array}$

$\begin{array}{lll}10.82107000 & -2.2100753000 & 14.248748000 \\ 10.714071000 & -2.794853000\end{array}$

$\begin{array}{lll}11.742438000 & -3.636263000 & 16.076800000\end{array}$

$\begin{array}{lll}12.597994000 & -3.826236000 & 15.429525000\end{array}$

$\begin{array}{lll}11.671779000 & -4.197951000 & 17.350308000\end{array}$

$\begin{array}{lll}12.479361000 & -4.840004000 & 17.705323000\end{array}$

$\begin{array}{lll}10.572551000 & -3.933690000 & 18.174622000\end{array}$

$\begin{array}{lll}10.515908000 & -4.377804000 & 19.169730000\end{array}$

$\begin{array}{lll}9.544983000 & -3.101141000 & 17.719839000\end{array}$

$\begin{array}{llll}8.682315000 & -2.899599000 & 18.356467000\end{array}$

$\begin{array}{llll}9.613497000 & -2.531190000 & 16.448799000\end{array}$

$\begin{array}{llll}8.806767000 & -1.894819000 & 16.084211000\end{array}$

$\begin{array}{lll}9.577480000 & -3.500721000 & 10.780764000\end{array}$

$\begin{array}{lll}8.879221000 & -4.718091000 & 10.271551000\end{array}$

$\begin{array}{rrr}9.646040000 & -5.839658000 & 9.905114000 \\ 10.729741000 & -5.786761000 & 9.99048000\end{array}$

$\begin{array}{lll}10.729741000 & -5.786761000 & 9.999048000 \\ 9.02708000 & -6.92123000 & 9.23583700\end{array}$

\begin{tabular}{lll}
9.027081000 & -6.992123000 & 9.423583000 \\
\hline 9.632676000 & -7.853189000 & 9.136777000
\end{tabular}

\begin{tabular}{lll}
9.632676000 & -7.853189000 & 9.136777000 \\
\hline 7.634562000 & -7.044585000 & 9.305317000
\end{tabular}

$\begin{array}{lll}7.6326562000 & -7.044585000 & 9.305317000 \\ 7.15028000 & -7.947134000 & 8.929403000\end{array}$

$\begin{array}{lll}7.6350280000 & -7.947134000 & 8.929403000 \\ 6.864286000 & -5.93410900 & 9.667068000\end{array}$

$\begin{array}{llll}7.150280000 & -7.947134000 & 8.929403000 \\ 5.778112000 & -5.93410900 & 9.667068000\end{array}$

$\begin{array}{lll}5.778112000 & -5.967861000 & 9.571920000 \\ 7.480068000 & -4.777004000 & 10.141295000\end{array}$

$\begin{array}{lll}7.780068000 & -4.777004000 & 10.141295000 \\ 6.879119000 & -3.907522000 & 10.40909600\end{array}$

$\begin{array}{rrr}6.879119000 & -3.907522000 & 10.409096000 \\ 10.553557000 & -0.272613000 & 9.255751000\end{array}$

$\begin{array}{rrr}10.553557000 & -0.272613000 & 9.255751000 \\ 11.954154000 & 1.169296000 & 11.946987000\end{array}$

$\begin{array}{lll}11.7702415000 & -1.740415000 & 9.881199000\end{array}$

$\begin{array}{lll}12.702415000 & -1.740415000 & 9.881199000 \\ 13.362015000 & -1.058342000 & 12.433945000\end{array}$

$\begin{array}{lll}13.362015000 & -1.058342000 & 12.433945000 \\ 12.077248000 & -1.051092000 & 9.008198000\end{array}$

$\begin{array}{lll}12.077248000 & -1.05352000 & 9.008198000 \\ 12.706964000 & -0.935414000 & 7.664021000\end{array}$

$\begin{array}{lll}12.766934000 & -0.935414000 & 7.664021000 \\ 13.648391000 & -1.902078000 & 7.264673000\end{array}$

$\begin{array}{lll}13.683772000 & -2.720746000 & 7.2643218000\end{array}$

$\begin{array}{rrr}14.260551000 & -1.814982000 & 6.016071000\end{array}$

$\begin{array}{lll}14.988188000 & -2.570453000 & 5.715802000\end{array}$

$\begin{array}{lll}13.944759000 & -0.761541000 & 5.151464000\end{array}$

$\begin{array}{lll}14.428793000 & -0.691236000 & 4.176063000\end{array}$

$\begin{array}{lll}13.011821000 & 0.204678000 & 5.541968000\end{array}$

$\begin{array}{llll}12.770727000 & 1.033524000 & 4.874966000\end{array}$

$\begin{array}{llll}12.395834000 & 0.121684000 & 6.790019000\end{array}$

$\begin{array}{llll}11.684749000 & 0.885639000 & 7.105767000\end{array}$

$\begin{array}{lll}13.298599000 & 0.214346000 & 12.469550000\end{array}$

$\begin{array}{lll}14.498157000 & 0.941512000 & 12.969436000\end{array}$

$\begin{array}{llll}15.763669000 & 0.337620000 & 12.854255000\end{array}$

$\begin{array}{lll}15.841260000 & -0.649343000 & 12.400350000\end{array}$

$\begin{array}{lll}16.903283000 & 1.004093000 & 13.300850000\end{array}$

$\begin{array}{lll}17.881714000 & 0.533507000 & 13.194429000\end{array}$

$\begin{array}{lll}16.793529000 & 2.272987000 & 13.879779000\end{array}$

$\begin{array}{lll}17.686278000 & 2.791955000 & 14.232046000\end{array}$

$\begin{array}{lll}15.537291000 & 2.874305000 & 14.008394000\end{array}$

$\begin{array}{llll}15.445841000 & 3.858664000 & 14.469653000 \\ 14.394658000 & 2.215947000 & 13.55543000\end{array}$

$\begin{array}{lll}14.394658000 & 2.215947000 & 13.555430000 \\ 13.412080000 & 2.674603000 & 13.667935000\end{array}$

$\begin{array}{llll}8.163268000 & 1.670382000 & 1.68235000\end{array}$

\begin{tabular}{lll}
8.163268000 & 1.670382000 & -11.582236000 \\
\hline
\end{tabular}

\begin{tabular}{llll}
6.394188000 & 3.474832000 & 11.576128000 \\
\hline 9.070365000 & 2.651975000 & 13.539955000
\end{tabular}

$9.522707000 \quad 3.088503000-10.247966000$

$6.702713000 \quad 3.896570000 \quad 13.447406000$

$\begin{array}{llll}6.702713000 & 3.885310000 & 13.447406000 \\ 7.680492000 & 4.839210000 & 11.079485000\end{array}$

$\begin{array}{llll}7.68502000 & 4.852192000 & 1.079485000 \\ 7.757300000 & 3.1178241000\end{array}$

$\begin{array}{lll}7.7876354000 & 4.213757000 & 15.473986000\end{array}$

$\begin{array}{lll}7.87691894000 & 5.332452000 & 15.810737000\end{array}$

$\begin{array}{lll}6.412561000 & 5.745733000 & 15.066960000\end{array}$

$\begin{array}{llll}6.412561000 & 5.745733000 & 15.066960000 \\ 7.194840000 & 5.910356000 & 17.074666000\end{array}$

$\begin{array}{lll}6.589323000 & 6.783449000 & 17.321850000\end{array}$

$\begin{array}{lll}8.075138000 & 5.376325000 & 18.021581000\end{array}$

$\begin{array}{llll} & & 8.5753 & \\ \mathrm{H} & 8.154247000 & 5.829193000 & 19.011167000\end{array}$ 


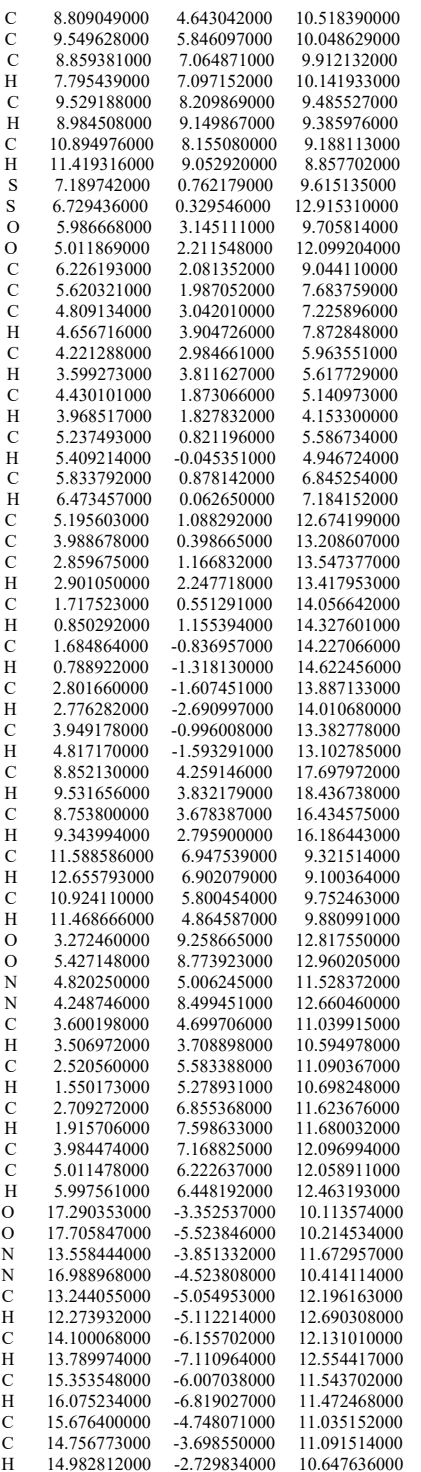

